\title{
Nasal prongs in the detection of sleep-related disordered breathing in the sleep apnoea/hypopnoea syndrome
}

\author{
E. Ballester*, J.R. Badia*, L. Hernández*, R. Farré**, D. Navajas**, J.M. Montserrat*
}

Nasal prongs in the detection of sleep-related disordered breathing in the sleep apnoeal hypopnoea syndrome. E. Ballester, J.R. Badia, L. Hernández, R. Farré, D. Navajas, J.M. Montserrat. CERS Journals Ltd 1998.

ABSTRACT: Conventional systems to monitor oronasal flow in sleep studies have traditionally relied on a thermistor signal. Our study was designed to verify whether nasal prongs (NP) connected to a pressure transducer could improve respiratory events detection in patients with sleep apnoea/hypopnoea syndrome (SAHS) compared to traditional systems.

Sleep episodes from a $2 \mathrm{~h}$ conventional polysomnographic record plus NP signal obtained at random from eight patients (age: mean $( \pm \mathrm{SD}) 53( \pm 12)$ yrs; body mass index $(\mathrm{BMI}): 29( \pm 6) \mathrm{kg}^{-\mathrm{m}^{-2}}$; apnoea/hypopnoea index (AHI): 27( \pm 20$)$ events'h ${ }^{-1}$ ) were identified and used for analysis. An abnormal change in the pattern of any of the respiratory or neurological variables occurring during the observation period was defined as an episode. Each episode was registered and scored with concomitant scoring of the remaining variables. According to the episode definition three different profiles were established: 1) periods of reduction of ventilation in either variable without an arousal or cyclical desaturation, named nonpathological episode (NPE); 2) an idiopathic or nonrespiratory arousal (IA); and 3) a true respiratory event (TRE) defined as reduction or absence of flow demonstrated by either thermistor, thoraco-abdominal bands or NP accompanied by cyclical desaturation and/or arousal. For each TRE, its detection by thermistor, thoraco-abdominal bands or NP was established.

A total of 877 sleep episodes were observed (42 NPE, 30 IA and 805 TRE). When compared to single or combined thermistor and bands approach, NP had the highest respiratory events detection rate, $779(96.8 \%)$ versus 673 events $(83.6 \%)$, respectively. Detection of respiratory-related arousals was also improved by NP and only $3 \%$ could account for mouth breathing respiration.

It is concluded that nasal prongs improve the detection of respiratory events in patients with sleep respiratory disorders.

Eur Respir J 1998; 11: 880-883.
*Servei de Pneumologia i Al.lèrgia Respiratòria, Departament de Medicina, Hospital Clínic, and **Laboratori de Biofísica Bioenginyeria, Universitat de Barcelona, Barcelona, Spain.

Correspondence: J.M. Montserrat Servei de Pneumologia i Al.lèrgia Respiratòria

Hospital Clínic

Villarroel 170

08036 Barcelona

Spain

Fax: 3432275454

Keywords: Airflow limitation

nasal cannule

respiratory events

sleep disordered breathing

thermistor

Received: April 291997

Accepted after revision January 161998

Supported by grants SEPAR 96, Fondo de Investigaciones Sanitarias (FIS) 95/236, Comisión, Interministerial de Ciencia y Tecnología (CICYT): SAF 96-0076 and from the Comissionat per a Universitats i Recerca (1995 SGR00446).
Conventional sleep studies are aimed to diagnose sleep disorders by assessment of respiratory patterns and nocturnal events. Changes in nasal and mouth airflow ventilation are currently detected and measured by thermally sensitive devices: thermistors [1]. Indirect parameters such as thoracic and abdominal movements measured with bands have also proven to be reliable [2]. Depending on the qualitative changes in the thermistor signal together with the thoraco-abdominal motion, apnoeas and hypopnoeas are routinely defined. Although all methods and devices used to monitor respiratory events do fairly well when scoring apnoeas, precision and reliability are specially required when considering hypopnoeas, known to be clinically as relevant as apnoeas but otherwise submitted to certain nonstandardized definition and thus, not infrequently overlooked [2, 3]. Hence, an ongoing controversy exists on how to define hypopnoea [4]. Few authors have addressed this issue showing that a definition based on a reduction on thoraco-abdominal movement provides a better correlation with other sleep variables than that based on thermistors [2]. In addition, upper airway resistance syndrome
(UARS) has made patent the need for newer devices to noninvasively detect partial airflow limitation of upper airways. Recently, a nasal prongs (NP) device connected to a pressure transducer has been suggested as a useful alternative to monitor respiratory events in patients with sleep apnoea/hypopnoea syndrome (SAHS) patients due to its ability to measure and quantify nasal flow [5]. The main advantage of the device is the good dynamic response compared to thermistors thus making detection and interpretation more accurate. The present study was designed to analyse the capability of NP to recognize respiratory events in SAHS patients through its comparison with the conventional assessment.

\section{Materials and methods}

\section{Study subjects (table 1)}

The classical polysomnographic records plus NP of eight patients (seven males) screened for SAHS were chosen for further study. Mean $( \pm S D)$ data from these subjects 
Table 1. - Anthropometric characteristics, pulmonary function data and sleep variables with conventional polysomnography

\begin{tabular}{lcccccr}
\hline $\begin{array}{l}\text { Patient } \\
\text { No. }\end{array}$ & $\begin{array}{c}\text { Age } \\
\text { yrs }\end{array}$ & $\begin{array}{c}\text { BMI } \\
\mathrm{kg} \cdot \mathrm{m}^{-2}\end{array}$ & $\begin{array}{c}\text { NC } \\
\mathrm{cm}\end{array}$ & $\begin{array}{c}\text { FEV1 } \\
\%\end{array}$ & $\begin{array}{c}\text { AHI } \\
\text { events } \cdot \mathrm{h}^{-1}\end{array}$ & ESS \\
\hline 1 & 52 & 29 & 42 & 115 & 8 & 1 \\
2 & 62 & 27 & 40 & 114 & 28 & 14 \\
3 & 58 & 25 & 40 & 95 & 8 & 18 \\
4 & 52 & 28 & 40 & 109 & 56 & 6 \\
5 & 54 & 29 & 47 & 82 & 13 & 2 \\
6 & 34 & 33 & 42 & 95 & 47 & 1 \\
7 & 72 & 42 & 46 & 69 & 45 & 10 \\
8 & 42 & 22 & 32 & 125 & 11 & 4 \\
Mean & 53 & 29 & 41 & 101 & 27 & 7 \\
SD & 12 & 6 & 5 & 19 & 20 & 6 \\
\hline
\end{tabular}

BMI: body mass index; NC: neck circumference; FEV1: forced expiratory volume in one second; AHI: apnoea/hypopnoea index; ESS: Epworth sleepiness scale.

was: age 53( \pm 11$) \mathrm{yrs}$; body mass index (BMI), 29( \pm 6$) \mathrm{kg}$. $\mathrm{m}^{-2}$, apnoea/hypopnoea index (AHI), 27( \pm 19$)$ events $\cdot \mathrm{h}^{-1}$; forced expiratory volume in one second (FEV1), 100 $( \pm 18)$ $\%$ pred; and Epworth sleepiness scale (ESS): $9( \pm 8)$. A series of $2 \mathrm{~h}$ periods selected at random (excluding the first and last hour) from the full night polysomnographic records from these patients were analysed for respiratory sleep events detection. The Ethics Committee of our hospital approved the protocol of this study and all patients gave their informed written consent.

\section{Polysomnography setup}

Polysomnography (PSG) was performed in the usual manner plus the addition of NP connected to a pressure transducer. PSG included continuous monitoring of the electrooculogram (EOG), electroencephalogram (EEG), and chin electromyogram (EMG) for sleep staging according to standard criteria [7]. Arterial oxygen saturation $\left(\mathrm{Sa}_{2} \mathrm{O}_{2}\right)$ was measured continuously with a finger probe, using a pulse oximeter (504 Critical Care Systems Inc, Waukesha, WI, USA). Rib-cage and abdominal motion were monitored by piezoelectric bands placed over the thorax and abdomen (Res-Ez; Tm Bionic, Midlothian, VA, USA). Airflow was measured through thermistors and NP. Three thermistors linked to two independent channels, one nasal and another oral, were used. The two nasal thermistors were attached on each outlet of the NP and placed in each nostril. Oral thermistor was placed in front of the mouth. $\mathrm{NP}$ had a $40 \mathrm{~cm}$ length and were connected to a differential pressure transducer (Validyne $\mathrm{MP} 45, \pm 5 \mathrm{cmH}_{2} \mathrm{O}$; Validyne Co., Northridge, CA, USA), as previously published [5]. No square root transformation of NP signal was applied. All signals were recorded continuously on a polygraph (1A98 Nicolet, Madison, WI, USA).

\section{Sleep events: definitions and protocol}

A skilled technician (8 yrs experience, with former background in EEG discipline), unaware of the main goal of the protocol but with wide experience in respiratory variables (including NP flow interpretation), visually assessed the records. The technician was required to monitor separately each individual change in respiratory and neurological variables, so-called episode, examining at that point, whether the other variables signals changed or not. When scoring an episode was debatable or uncertain, two skilled sleep staff physicians resolved by consensus with the technician.

An episode was defined when a change in the pattern of any of the respiratory or neurological variables occurred during the observational period. All respiratory and neurological variables were identified and qualitatively scored for the presence or absence of any significant change. An NP episode was defined not only when there was a decrease in the flow signal but also when a sudden change in nasal flow morphology developed from a flow-limited (plateau-like) inspiratory pattern to a nonlimited (rounded) inspiratory flow. Apnoeas and hypopnoeas were defined according to commonly used clinical criteria of airflow cessation lasting $10 \mathrm{~s}$ or more for apnoeas and a $10 \mathrm{~s}$ or more period of discernible airflow reduction associated with an arousal and/or a cyclical $\mathrm{Sa}, \mathrm{O}_{2}$ dip for hypopnoeas. Apnoeas or hypopnoeas were considered together due to the same underlying pathophysiological significance [2]. Arousals were scored according to the American Sleep Disorders Association (ASDA) recommendations [6, 7] and microarousals were scored according to these same criteria, but with a duration of $1.5 \mathrm{~s}$ [8] in association with EMG activity.

According to the episode's definition, three different profiles were established: 1) periods of reduction of ventilation in any signal without an arousal or cyclical oxygen desaturation, named nonpathological episode (NPE); 2) an idiopathic or nonrespiratory arousal (IA); and 3) a true respiratory event (TRE), defined as a reduction or absence of flow demonstrated by either thermistor, thoraco-abdominal bands or NP accompanied by cyclical desaturation and/or arousal. For each true event, its detection by thermistor, thoraco-abdominal bands or NP was established.

\section{Statistical analysis}

Statistical analysis was performed with Statistical Package for Social Sciences v 6.1.3 for windows (SPSS Inc, Chicago, IL, USA). Statistics derived from frequencies, cross-tabulations and the measures of sensitivity as the index of efficacy were used when appropriate. Statistical significance was accepted at $\mathrm{p}<0.05$.

\section{Results}

In the $2 \mathrm{~h}$ period analysed we did not observe any loss of signal due to displacement of the cannula or secretions. A total of 877 sleep episodes were identified from the $2 \mathrm{~h}$ nonselected period from the eight patients' records, $84 \%$ of which were during non-rapid eye movement (REM) sleep and $16 \%$ during REM sleep. Episodes and TRE were scored as positive, negative or uncertain. Data on the overall number and frequency of sleep episodes registered $(n=877)$ are shown in table 2, left side. The data are further arranged to select those considered TRE $(n=805)$ (table 2, right side). The difference between episodes and TRE $(n=72)$ represents those nonpathological changes in ventilation plus the IA. Arousals were considered idiopathic when present without observed changes in monitored respiratory variables (thermistor, thoraco-abdominal bands, nasal flow or $\mathrm{Sa}_{\mathrm{a}} \mathrm{O}_{2}$ ). From the 877 episodes, 30 
Table 2. - Overall episodes and true respiratory events

\begin{tabular}{lccccccc}
\hline & \multicolumn{3}{c}{$\begin{array}{c}\text { Episodes* } \\
\text { (n=877) }\end{array}$} & & & \multicolumn{2}{c}{ True respiratory events } \\
& & & & & & & \\
& Positive & Negative & Uncertain & & Positive & Negative & Uncertain \\
\hline Arousal $(\mathrm{n}=877) \mathrm{n} \%$ & $655(75)$ & $222(25)$ & & & $625(78)$ & $180(22)$ & \\
Thermistor $(\mathrm{n}=877) \mathrm{n} \%$ & $503(57)$ & $261(30)$ & $113(13)$ & & $485(60)$ & $215(27)$ & $105(13)$ \\
T-A bands $(\mathrm{n}=876) \mathrm{n} \%$ & $695(79)$ & $101(12)$ & $81(9)$ & & $669(83)$ & $69(9)$ & $67(8)$ \\
Nasal prongs $(\mathrm{n}=877) \mathrm{n} \%$ & $821(94)$ & $35(4)$ & $21(2)$ & & $779(97)$ & $12(2)$ & $14(2)$ \\
\hline
\end{tabular}

T-A bands: thoraco-abdominal bands. *: overall episodes frequency (idiopathic arousals, nonpathological respiratory events and true respiratory events).

arousals $(3.4 \%)$ were considered physiological $(4.6 \%$ of arousals). Nonpathological ventilatory events $(n=42,4 \%)$ were considered when either changes in thermistor, thoraco-abdominal bands or nasal flow were not accompanied by arousal nor oxygen desaturation. These events should be considered as the false positive rate of the different respiratory variables.

RTE were thus considered after excluding physiological arousals and nonpathological respiratory events. All 805 respiratory events were considered for further analysis and recoded initially to positive (if a change was present in the variable) or negative (when unchanged or uncertain) giving strength to the evidence-based observation. From the 805 TRE, $779(96.8 \%)$ were detected by NP while $485(60 \%)$ and $669(83 \%)$ by thermistor and thoraco-abdominal bands, respectively. When NP was compared to the number of combined positive results in either thermistor or bands (673 events $(83.6 \%)$ were positive and 132 (16.4\%) negative), still more events were detected by NP. In addition, NP had the least number of events defined as uncertain. Figures 1 and 2 are two representative respiratory events of NP along with the remaining variables.

\section{Respiratory arousals detection}

As previous data are descriptive and defined true events include the variables under investigation, there is no "gold standard" to compare the three methods. In this study, we have selected respiratory arousals as the "gold standard" approach to compare the three methods of detection of changes in ventilation. This assumption or selection is based

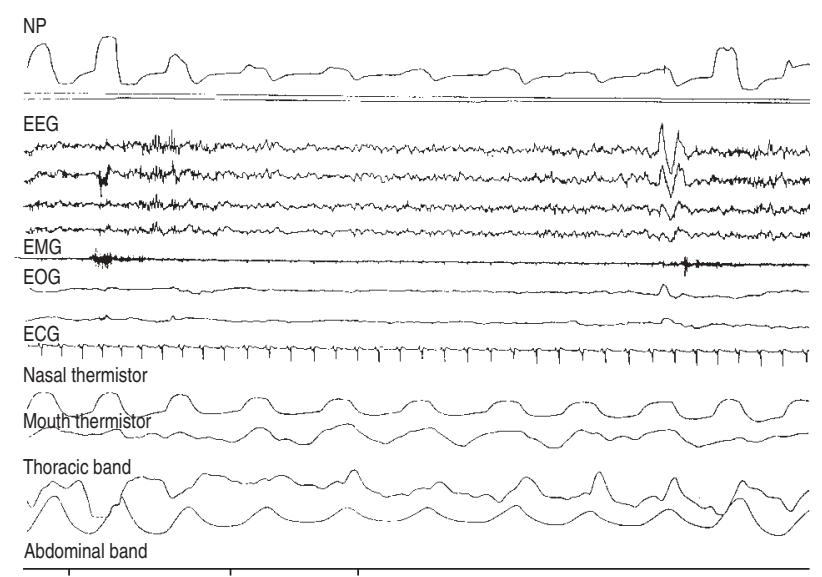

Fig. 1. - A representative polysomnographic view of the behaviour of nasal prongs (NP) during a hypopnoea between two arousals. Note the poor detection obtained through thermistors. A respiratory event could be missed if thermistor were the sole respiratory method for detection of airflow. EEG: electroencephalogram; EMG: electromyogram; EOG: electro-oculogram; ECG: electrocardiogram.

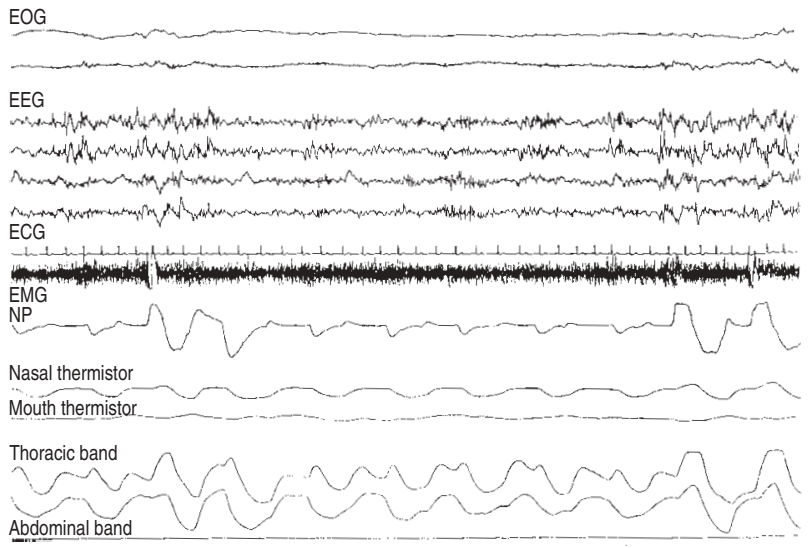

Fig. 2. - A representative polysomnographic view of the behaviour of nasal prongs (NP) during a hypopnoea between two arousals. Thermistors remain unchanged during arousal with partial inco-ordination of thoracic and abdominal bands. Relying only in thermistors, the airflow events could be missed. For definitions refer to figure 1 .

Table 3. - Frequency of respiratory arousal detection by the three devices and combined thermistor and thoracoabdominal (T-A) bands

\begin{tabular}{llrr}
\hline & Ventilation & \multicolumn{2}{c}{ Arousals } \\
\cline { 2 - 4 } & & Yes & No \\
\hline Thermistor & Reduced & 389 & 96 \\
& Normal & 236 & 84 \\
T-A bands & Reduced & 511 & 158 \\
& Normal & 114 & 22 \\
Thermistor plus & Reduced & 515 & 158 \\
T-A bands & Normal & 110 & 22 \\
Nasal prongs & Reduced & 603 & 176 \\
& Normal & 22 & 4 \\
\hline
\end{tabular}

Values are presented as absolute number.

on its close association with respiratory events but at the same time on its independence from them. As screening is the main purpose and detection its aim, sensitivity was used as the index of efficacy of the diagnostic tests. NP improved detection of respiratory-related arousals, 603 detected by NP compared to 389 and 511 for thermistor and thoraco-abdominal bands, respectively (table 3 ). As a result, a sensitivity of 62,81 and $96 \%$ was obtained for thermistors, thoraco-abdominal bands and NP, respectively. Other indices of efficacy were not analysed as scored uncertainties preclude their appropriate interpretation.

\section{Discussion}

Our study was designed to evaluate the introduction of recording of nasal flow (flow signal) through NP to the polysomnographic assessment of respiratory sleep events. In particular, we wished to evaluate to what extent nasal flow could assist in improving the detection of sleep events when combined with the commonly used conventional 
sleep detection variables. Thus, nasal flow through NP increased the number of respiratory events detected when compared to either thermistor or thoraco-abdominal bands or with the two techniques combined. In addition, NP was associated with a better detection rate of respiratory-related arousals. During the $2 \mathrm{~h}$ period analysed we did not observe any loss of signal.

Thermistors are the conventional systems to monitor nasal and mouth airflow in sleep studies [1]. However, it is well known that the signal obtained through thermistors usually employed in sleep studies has a poor timeresponse [9] and marked nonlinearity [10]. The result is a delay in the apparent timing of respiration in relation to the intrathoracic pressure changes (measured by an oesophageal balloon) thus limiting the accuracy of the procedure. Recently, we and others have proved that measurement of inspiratory flow characteristics is a reliable method to estimate the lowest oesophageal pressure during continuous positive airway pressure (CPAP) titration [11, 12]. In addition, the inability to detect airflow characteristics and the underestimation of actual flow makes thermistor a limited device for interpretation purposes.

NP have been suggested as a useful alternative to the thermistor to measure nasal flow [5]. Nevertheless, NP may present some problems due to its inability to detect mouth breathing during sleep. Most people breathe through the nose and thus potential advantages of measuring flow with NP would probably overcome this limitation. However, this limitation of the technique is clearly counterbalanced by a more accurate overall event detection rate. In addition, we have recently observed that the mean percentage of time that people breathe through the mouth when asleep is very small [13].

Because of their excellent dynamic response, NP should allow the analysis of both flow and its morphology. Moreover, an additional advantage over the indirect thermistor output signal is the detection of snore which is an airflow-related phenomenon that produces an easy to identify high frequency vibration that has a considerable interest in sleep studies [14]. Partial airflow limitation (inspiratory flow contour flattening) and the snoring-related vibrations are best observed by the flow morphology. Thus, flow measured with NP provides valuable additional information that constitutes a substantial advantage over the thermistor signal. Recently, we have established the background for NP use for estimating nasal flow [5].

In conclusion, our results suggest that the use of NP signal is a simple procedure to accurately assess airflow during routine sleep studies. The advantages of this device are: 1) NP are easily tolerated during the period studied; 2 ) the high respiratory detection rate of NP increases the accuracy of measurements; and 3) as opposed to thermistors, the excellent time response of NP should allow to precisely detect or correlate for each different part or event within the breathing cycle making physiopathological studies more reliable. In addition, snoring can be easily detected from the NP signal as an inspiratory as well as expiratory phenomena (not seen by thermistor or bands). Further studies are needed to evaluate the clinical utility of NP in assessing snoring.

The role of NP as a tool in the sleep laboratory is not yet established. It is our belief that, if this technique confirms its promise, NP will be routinely introduced in clinical practice. From our study NP should be considered a valuable tool which offers complementary information to the other devices used and can be applied without discomfort even when added to the thermistor which is actually our routine setup.

Because of the increased detection of respiratory events through NP, a further consequence could be expected. We know that a special concern in sleep studies is the frequent dissociation between indices relating to respiratory disturbances and the clinical symptoms. From our study it is clear that the number of sleep events scored as respiratory increased. This would provide us with different numbers or indices of respiratory disturbances to associate with clinical symptoms and perhaps better correlation could be found when evaluating sleep studies, in particular, in those patients with UARS or in nonsevere SAHS patients.

\section{References}

1. Parisi, RA, Santiago TV. Respiration and respiratory function: Technique of recording and evaluation. In: Chokroverty S, ed. Sleep Disorders Medicine. Basic Science, Technical Considerations and Clinical Aspects. Boston, Butterworth-Heinemann, 1994; pp. 127-133.

2. Gould GA, Whyte KF, Rhind GB, et al. The sleep hypopnea syndrome. Am Rev Respir Dis 1988; 137: 895-898.

3. Bliwise D, Bliwise NG, Kraemer HC, Dement WC. Measurement error in visually scored electrophysiological data: respiration during sleep. J Neurosci Methods 1984; 12: 429-456.

4. Moser N, Phillips B, Berry D, Harbison L. What is hypopnea anyway? Chest 1994; 105: 426-428.

5. Montserrat JM, Farré R, Ballester E, Felez MA, Pastó M, Navajas D. Evaluation of nasal prongs for estimating nasal flow. Am Respir Crit Care Med 1997; 155: 211-215.

6. U.S. Public Health Service. Rechtschaffen A, Kales A, eds. A Manual of Standardized Terminology, Techniques and Scoring System for Sleep Stages of Human Subjects. U.S. Government Printing Office, Washington, DC., 1966.

7. ASDA report. EEG arousals: scoring rules and examples. Sleep 1992; 15: 174-184.

8. Mathur R, Douglas NJ. Frequency of EEG arousals from nocturnal sleep in normal subjects. Sleep 1995; 18: 330333.

9. Xiong C, Sjoberg BJ, Sveider P, Ask P, Loyd D, Wranne B. Problems in timing of respiration with nasal thermistor technique. J Am Soc Echocardiogr 1993; 6: 210-216.

10. Farré R, Montserrat JM, Rotger M, Ballester E, Navajas D. Accuracy of thermistors and thermocouples as flow measuring devices for detecting hypopneas. Eur Respir $J$ 1998; 11: 179-182.

11. Montserrat JM, Ballester E, Olivi H, et al. Time-course of stepwise CPAP titration. Behaviour of respiratory and neurological variables. Am J Respir Crit Care Med 1995; 152: 1854-1859.

12. Condos R, Norman RG, Krishnasamy I, Peduzzi N, Goldring RM, Rapoport DM. Flow limitation as a noninvasive assessment of residual upper-airway resistance during continuous positive airway pressure therapy of obstructive sleep apnea. Am J Respir Crit Care Med 1994; 150: 475-480.

13. Hernández L, Ballester E, Reolid A, Fornas C, Rodriguez Roisin R, Montserrat JM. Breathing route detected by conventional devices (thermistor). Am J Respir Crit Care Med 1997; 155: A130.

14. Lugaresi E, Cirignotta F, Montagna P, Sforza E. Snoring: pathogenic, clinical and therapeutic aspects. In: Kryger MH, Roth T, Dement WC, eds. Principles and Practice of Sleep Medicine. 2th ed. Philadelphia, W.B. Saunders, 1994; pp. 621-629. 